At the operation a large exostosis was found growing from the posterior wall of the meatus, and the anterior and posterior walls were adherent. The exostosis was chiselled away, and the tympanum and mastoid cells were found packed with cholesteatomatous masses, which were removed. The marked symptoms of thrombosis of the cavernous sinus rendered it probable that the transverse sinus would also be affected (by extension along the petrosal sinus), and it was therefore exposed, but found to be normal. The exophthalmos increased, and the patient died four days after operation. P'ost-mortem: A large extradural abscess overlay the discoloured but intact tegmen tympani, extending as far as the cavernous sinus, which was full of broken-down ichorous clots. The carotid and the roots of the fifth nerve were bathed in pus. Above the cavernous sinus there was a patch of purulent meningitis; the retrobulbar tissue was suppurating; the petrosal and transverse sinuses were normal. Thus thrombosis of the cavernous sinus occurs from suppurative disease of the middle fossa, though no doubt it is rare.

Monatschrift fïr Ohrenheilkunde, March, 1900.

William Lamb.

\title{
Abstracts.
}

\section{MOUTH, Etc.}

Minerbi, Dr. C. (Florence).-External Palpation of the Tonsillar Region. “ Bolletino," Florence, April, 1901. (Review by Dr. V. Grazzi.)

As it sometimes happens that it is impossible to examine the fauce of a child, it is very important to have some external sign which will inform us of the existence of an acute affection of the fauces.

According to the author, this sign consists in swelling, which occurs early, especially in diphtheria, and appears in a point corresponding to the superior triangle of the neck, situated between the angle of the jaw, the anterior margin of the sterno-mastoid, and the greater cornu of the hyoid. The author states that in the course of twenty years' experience there is swelling of the amygdalic glands of Chassaignac situate in this part, always from the commencement of fever, and this not only in diphtheria, but in all the acute tonsillar affections of children.

One must beware of confounding the glands of Chassaignac with another group, rather more superficial and mobile, which occurs in the superficial fascia immediately below the angle of the jaw, and which, according to the author, receive the lymphatics of the gum and second bicuspid of the same side.

Some have thought that this swelling might be the inflamed tonsil itself, felt from outside, but the author has excluded this by demonstrations on the dead body, and established that the swelling is due to the engorgement of the amygdalic glands of Chassaignac. 
A day or two from the occurrence of fever from a tonsillar affection, swelling takes place in another group of glands which the author calls the inferior amygdalic glands, situated immediately external to the corresponding greater cornu of the hyoid. This swelling disappears with the cessation of fever, and before that of the glands of Chassaignac. Minerbi, after most careful observation, concludes that the amygdalic glands are anatomically separated from the other lymphatics, and describes clearly the nature of this separation. James Donelan.

Prota, Dr. G. (Naples).-Fibroma of the Tonsil. "Archiv. Ital. de Laringologia," January, 1901.

Dr. Prota describes the history and symptoms of this affection at considerable length, and gives an extensive bibliography. The case was that of a woman aged fifty who had suffered frequently from tonsillitis. There was a large pedunculated tumour curved "like an eagle's beak," and growing from a base about 3 centimetres in diameter at the upper part of the left tonsil. There was no pain, and no glandular enlargement. The growth was snared by Professor Massei under cocaine, and was found to have the ordinary structure of a fibroma. James Donelan.

\section{NOSE, Etc.}

Cholewa, Dr. (Cassel).-Why do Nasal Polypi recur? " Monatschrift für Ohrenheilkunde," March, 1900.

The author differs from Hermann, who thinks polypi are always secondary to irritation, generally from pus; and also from Hajek, who thinks the irritation generally starts from the surface of the mucosa, and that the bone-changes are secondary. He directs attention to the formation of new bone occurring after influenza, under the periosteum, which is thickened and vascular, and may contain tiny abscesses and necroses. More frequent than large subperiosteal abscesses such as occur after enteric are supericial erosions of the surface of the bone. The periosteum is thickened and vascular, and easily separable as the result of a kind of subacute osteo-myelitis-rarifying ostitis. In calling this disease caries, Woakes only used the nomenclature of his time, which had not yet distinguished between the specific tuberculous ulcer of bone, true caries, and the eroding ostitis of other infectious diseases. Rarifying ostitis is not the result of inflammation of the mucosa, but rather it is the result or final stage of a long-continued inflammatory process of the lower periosteum.

William Lamb.

\section{Garrow, A. E.-Todent Ulcer of the Nose. "Montreal Medical Journal," January, 1901.}

The patie $t$ was a woman, seventy-one years of age. Twenty-two years previously three small nodules developed in the inner corner of the right eye. These broke down and gradually ulcerated, and were followed by a new crop of tubercles on the other side. The sore covered the whole right side of the nose from the angle of the orbit, and extended over to the left side. It was dry, covered with scab, and exuded no fluid. The edge was infiltrated, though not much elevated, and there were no surrounding nodules. It was not painful.

The treatment consisted of removal by the scalpel of the tissues 